\title{
Unilateral Cryptorchidism in a Newborn with Chronic Inflammation
}

\author{
Lucas Borje Cea ${ }^{1 *}$ and Miguel Borje Ranquiman ${ }^{2}$ \\ ${ }^{1}$ Facultad de Medicina y Ciencia, Universidad San Sebastián, Chile \\ ${ }^{2}$ Complejo asistencial Dr. Victor Ríos Ruiz, Chile
}

*Corresponding author: Lucas Borje Cea, Facultad de Medicina y Ciencia, Universidad San Sebastián, Concepción, Chile.

To Cite This Article: Lucas Borje Cea, Miguel Borje Ranquiman, Unilateral Cryptorchidism in a Newborn with Chronic Inflammation. Am J Biomed Sci \& Res. 2021 - 12(4). AJBSR.MS.ID.001763. DOI: 10.34297/AJBSR.2021.12.001763.

Received: March 31, 2020; Published: 制 April 08, 2021

\begin{abstract}
Cryptorchidism is a rare condition often considered to be a pathological sign of and underlying disorder. We report the case of a newborn with no palpable right testicle in the scrotum, who underwent surgical removal of the remnant tissue, after imaging studies. Pathological analysis found unusual characteristics of the sample, requiring further immunohistochemistry analysis that ultimately revealed necrosis and chronic inflammation. Although these findings could no clarify the cause of cryptorchidism, physician must be aware that monitoring the patient could be necessary to provide early adequate health care.
\end{abstract}

Keywords: Cryptorchidism; Unilateral; Inflammation; Newborn

Abbreviation: PLAP: Placental Alkaline Phosphatase

\section{Introduction}

Cryptorchidism is described as a total or partial failure of testicles descent from the scrotal sack, being detected in around $1 \%$ of children at 1 year of life [1]. It is not considered a disease on its own, but rather a sign of other pathological conditions, such as Prader-Willi syndrome, Kallman syndrome and hypopituitarism, among others. For that reason, is important to determine the cause of cryptorchidism, to provide an early assessment of the patient health condition, through the study of several hormones and/or genes that might be affected [2]. We report the case of a newborn with unilateral cryptorchidism, whose histopathological analysis of the remnant testicle revealed necrosis and chronic inflammation signs.

\section{Case Report}

A 1-day old male newborn presents himself with a failure of the right testicle descent, not unidentifiable by palpation. By contrast, the left testicle is in position, with normal consistency and no other anomalies are detected at simple examination. We proceed to analyze the right testicle through echography, founding an inflamed and atrophic mass in the inguinal canal. A decision was made to surgically remove the mass after confirming severe damage. Macroscopic examination reveals an irregular nodular fragment, size of $10 \times 8 \times 4 \mathrm{~mm}$, accompanied by spermatic cord, $4 \mathrm{~cm}$ long $\mathrm{x} 1 \mathrm{~mm}$ diameter. Dissection of the sample allowed the recognition of a $4 \mathrm{~mm}$ cavity with brownish material and another yellow and oval cyst of $3 \mathrm{~mm}$. Microscopic analysis found nodular fibrosis, central necrosis, and calcified material, suggesting testicular rests. Fragments of fibroconnective tissue and deferent conduct were structurally conserved. To clarify the etiology of the findings, immunohistochemistry analysis was performed on the samples using antibodies against CD117 (staining tumor cells), inhibin (staining Sertoli and Leydig cells), placental alkaline phosphatase PLAP (staining testicular germ-cell neoplasm) and D2-40 (staining lymphatic endothelium and neoplastic cells). The samples were positive only for inhibin, consistent with atrophic testicular tissue (Figure 1). 


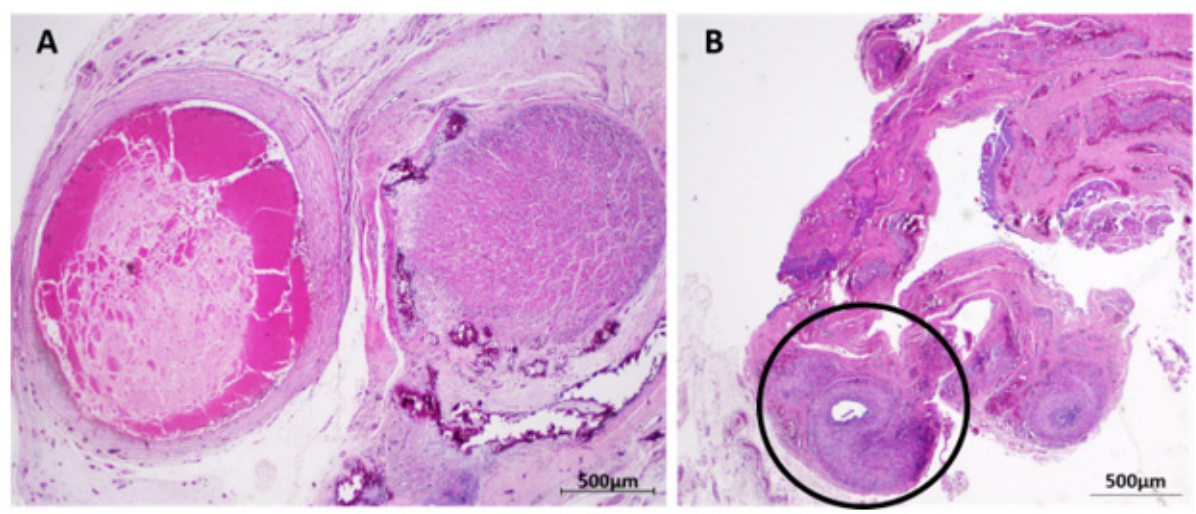

Figure 1: Histopathological analysis of the atrophic testicular mass. A: At the right side of the image a nodular structure of necrotic seminiferous tubules can be seen, with scant lymphoplasmacytic inflammatory infiltrate, foreign body-type giant cells and dystrophic-type calcification foci. At the left side of the image, necrotic nodule surrounded by thick bands of eosinophilic collagen fibers. B: Spermatic chord. Fragment of fibroconnective and adipose tissue with moderate fibrosis in which a transversal section of the vas deferens can be recognized.

Because of the severe damage found in the histopathological analysis, intrauterine torsion injury is unlikely to be the possible cause, which should be detected as a more acute presentation. It is hypothesized that the cryptorchidism es due chronic inflammation. Unfortunately, no other tests could be performed on the infant to determine the cause of this inflammation, and follow-up of the baby or his mother was not possible.

\section{Discussion}

Despite the scarce knowledge about the causes and physiopathology of cryptorchidism, there are therapies that seek to position the testicle anatomically, to preserve its functionality satisfactorily and prevent testicular cancer. However, these therapies must begin as soon as possible and must be chosen properly to ensure no complications by mechanical damage or inadequate temperature arise [3]. In this case, the non-palpable testicle was severely damaged, showing signs of necrosis and inflammation of undetermined cause, so It had to be removed. The inability to complete the diagnostic procedure with other test and follow ups, unfortunately prevent us to prepare the family for other complications that might arise during the infant development, as well as rule out a major underlying health condition. Other studies have shown that in unilateral cryptorchidism, the contralateral testicle exhibit different histological alterations at the age of two years old [1], warning the need to keep close monitoring of the patient to provide early adequate health care.

\section{Acknowledgement}

The authors would like to thank Dr. Karina Oyarce for helping us with the manuscript and the Healthcare Complex Dr. Victor Ríos Ruiz.

\section{References}

1. Kumar V, Abbas A, Aster J (2015) Robbins y Cotran patologia estructural y funcional (9th Edn), ELSEVIER, Barcelona, España, pp. 1059-1060.

2. Blanco S, Gottlieb S, Grinspon R, Rey R (2015) Criptorquidia: desde la embriología al tratamiento. Médicas UIS 28(3): 371-380.

3. https://ri.conicet.gov.ar/bitstream/handle/11336/103120/ CONICET_Digital_Nro.377045f7-73b0-432a-87d0-66b61fef85b8_A. pdf? sequence $=2 \&$ isAllowed $=y$ 